\title{
« Notre cadavre quotidien », représentations littéraires de la mort et de la violence en Amérique centrale
}

\begin{abstract}
Avertissement
Le contenu de ce site relève de la législation française sur la propriété intellectuelle et est la propriété exclusive de l'éditeur.

Les œuvres figurant sur ce site peuvent être consultées et reproduites sur un support papier ou numérique sous réserve qu'elles soient strictement réservées à un usage soit personnel, soit scientifique ou pédagogique excluant toute exploitation commerciale. La reproduction devra obligatoirement mentionner l'éditeur, le nom de la revue, l'auteur et la référence du document.

Toute autre reproduction est interdite sauf accord préalable de l'éditeur, en dehors des cas prévus par la législation en vigueur en France.
\end{abstract}

\section{revues.org}

Revues.org est un portail de revues en sciences humaines et sociales développé par le Cléo, Centre pour l'édition électronique ouverte (CNRS, EHESS, UP, UAPV).

Référence électronique

Sergio Coto-Rivel, « « Notre cadavre quotidien », représentations littéraires de la mort et de la violence en Amérique centrale », Amerika [En ligne], 12 | 2015, mis en ligne le 03 juillet 2015, consulté le 12 mai 2016. URL http://amerika.revues.org/6372 ; DOI : 10.4000/amerika.6372

Éditeur : LIRA-Université de Rennes 2

http://amerika.revues.org

http://www.revues.org

Document accessible en ligne sur :

http://amerika.revues.org/6372

Document généré automatiquement le 12 mai 2016

(c) Tous droits réservés 


\title{
Sergio Coto-Rivel
}

\section{« Notre cadavre quotidien », représentations littéraires de la mort et de la violence en Amérique centrale}

\section{Introduction. Espaces de violence}

Qu'elle ait recours à l'ironie, à un humour noir débordant ou à un réalisme cru, la littérature centraméricaine contemporaine reprend avec force la violence dans laquelle elle fut conçue dans les deux dernières décennies, mettant l'accent non seulement sur la dénonciation $\mathrm{du}$ contexte de peur, mais aussi sur la naturalisation d'un état dont les habitants acceptent de plus en plus, et de manière quotidienne, le fléau qui s'abat sur les grandes villes de la région. De nombreux exemples de la littérature contemporaine dépeignent des espaces de violence croissante à de différents niveaux de la société. Les arrière-goûts de la guerre pour d'aucuns (Nicaragua, Guatemala, Salvador) et la criminalité croissante (particulièrement au Costa Rica et au Panama pour les décennies précédentes) aiguisent le climat de tension de la population qui doit se confronter jour après jour à cette réalité. En plus de la littérature, les arts visuels reprennent fortement aussi ces impressions ${ }^{1}$. La banalisation de la violence se fait toujours plus évidente dans les sociétés de l'Isthme. Pour preuve, les médias relatent le thème de manière constante, provoquant la peur au quotidien ${ }^{2}$.

Plus de cinq ans après la signature des derniers accords de paix, la région centraméricaine souffre toujours d'un climat de violence - en particulier le Salvador, le Guatemala et le Honduras - qui fait penser à un état de guerre constante. Les victimes de la délinquance et du narcotrafic se comptent comme l'on recensait les victimes des affrontements entre guérillas et armée ${ }^{3}$. L'attention que l'on a prêtée à l'Amérique centrale au niveau mondial durant les années 80 décrut significativement après la signature des accords. Ce fut précisément cette mise en lumière en temps de conflit qui donna à l'Isthme cette réputation de région dangereuse. Dix ans plus tard, l'intérêt a repris. Et malgré la grande différence du contexte politique, les résultats se résument de nouveau à la mort et à la pauvreté. Cependant, cette fois-ci, l'intérêt se focalise sur le trafic de drogue qui se développe intensément dans ce couloir menant aux États-Unis, ainsi que sur l'organisation en augmentation de bandes de jeunes (que l'on appelle maras, en particulier au Guatemala, au Honduras et au Salvador) et enfin sur la libre circulation des armes à feu comme les trois principales raisons de violence. Tout cela au sein d'un engrenage de causes et de conséquences impliquant les vols quotidiens, les agressions de rue et la violence sexuelle et domestique. Le rapport de la Banque mondiale, Crimen y violencia en Centroamérica, un desafío para el desarrollo ${ }^{4}$, ouvre la discussion avec une comparaison claire des niveaux de criminalité dont souffre la région :

\begin{abstract}
El crimen y la violencia constituyen el problema clave para el desarrollo de los países centroamericanos. En tres países — El Salvador, Guatemala y Honduras — los índices de crimen y violencia se encuentran entre los tres más altos de América Latina. En los demás países de la región - Costa Rica, Nicaragua y Panamá - los niveles de crimen y violencia son significativamente menores, pero un aumento sostenido de los índices de violencia en años recientes es motivo de preocupación. Existen razones para ello. A fin de poner en contexto la magnitud del problema, la población total de Centroamérica es aproximadamente la misma que la de España; sin embargo, en 2006, España registró 336 asesinatos (es decir menos de uno por día) y Centroamérica registró 14.257 asesinatos (es decir casi 40 por día). (Banco Mundial, 2011 : II)
\end{abstract}

La citation présente clairement le contexte. Cependant, les chiffres et les statistiques ne suffisent jamais à analyser en profondeur et à comprendre les dynamiques sociales en marche derrière toutes ces situations de violence. Les chiffres nous donnent une idée comparative et créent en même temps la sensation, chez les récepteurs (lecteurs des rapports et surtout téléspectateurs des journaux télévisés), d'être confrontés au crime de manière continuelle. C'est dans cette perception, dans laquelle se jouent les situations politiques, que se déterminent 
les principales tendances électorales pour convaincre le peuple qu'une plus grande attention et une action gouvernementale directe sur le problème de la violence seront accordées.

Une des sources importantes de violence mentionnée ci-dessus, la violence juvénile, a marqué considérablement les études, non seulement en Amérique centrale, mais aussi dans les universités nord-américaines et européennes. Le cas des maras $^{5}$ épouvante et fascine à la fois les critiques culturels, étant un phénomène de grande envergure impliquant une dévotion intense au sein d'un monde de violence extrême ${ }^{6}$. Les affrontements entre gangs, majoritairement composés d'hommes, sont la cause de nombreux meurtres jour après jour, faisant de leurs membres les principales victimes et auteurs d'homicides dans les statistiques de chaque pays. Nous remarquons ainsi que c'est la population juvénile qui se retrouve le plus lésée (des jeunes âgés de quinze à vingt-cinq ans en majorité selon le rapport). Les villes centraméricaines en croissance rapide, notamment celles qui ont accueilli une importante population urbaine suite aux conflits armés, sont celles qui souffrent le plus de la violence en raison du manque de planification urbaine dans des espaces publiques extrêmement détériorés. À cela s'ajoute le chômage des jeunes, au sein d'un climat de violence déjà installé avec son système de normes et de valeurs justifiant les actes illicites (Banco Mundial, 2011 : 19). L'inscription à l'enseignement secondaire est très faible, favorisant la grande tendance au chômage, surtout dans des zones de grande inégalité salariale. Les facteurs d'ordre relationnel ne peuvent être mis de côté puisque, dans de nombreux cas, les compagnons et amis membres des bandes juvéniles deviennent l'unique soutien moral et économique de ces jeunes en difficulté. Comme nous le voyons, les causes sont multiples et le système au sein duquel elles agissent se révèle de plus en plus complexe et difficile à contrôler. Les statistiques ne suffisent pas à tirer des conclusions déterminantes quant à la portée du problème de la violence en général.

Face à cette situation, nous nous demandons pourquoi l'Isthme centraméricain est confronté à un quotidien d'actes violents qui le saignent. Le rapport de la Banque mondiale avance l'hypothèse selon laquelle les accès de violence seraient une cause directe des conflits armés, lesquels non seulement sont une manière de réagir à l'injustice ou au mécontentement, mais aussi, d'un point de vue pratique, facilitent dans la région la circulation incessante d'armes, et ce de manière illégale. Or, ces évidences quant aux guerres internes ne peuvent expliquer totalement les faits des dernières décennies, en particulier la recrudescence des crimes dans des pays comme le Costa Rica ou le Panama, qui ne vécurent pas les affrontements des pays voisins de la même façon. Pourtant, les indices de violence continuent d'y augmenter.

Les coûts directs et indirects de la violence en Amérique centrale sont payés durement et de manière inégale par les pays de la région, puisque les effets, comme nous le voyons, n'en ont pas été les mêmes (il suffit de comparer les données du Guatemala et du Costa Rica par exemple). Les efforts pour mener une lutte contre le narcotrafic ne minimaliseront probablement pas le problème, cependant, cette dernière fut l'une des principales batailles menées par les gouvernements dans le but de promouvoir leurs programmes de combat contre la violence en général.

Il est donc nécessaire de nous demander si nous pouvons nous référer uniquement à des facteurs géopolitiques mettant en évidence les jeux de pouvoir entre les pays du Nord et leur interventionnisme dans l'hémisphère sud, particulièrement au sujet du trafic de drogue ou des opérations militaires antérieures et du financement des guérillas pour expliquer la recrudescence et la permanence des actes de violence. L'importance de ces facteurs saute immédiatement aux yeux dans toutes les analyses de la région réalisées par d'importantes institutions internationales. Cependant, la structuration des discours de violence, ainsi que ses causes les plus intrinsèques, ne peut être analysée ni comprise ni synthétisée ni encore moins se résumer à l'énumération de trois ou quatre facteurs visibles et à la fois choquants de par leur force symbolique.

\section{Claudia Hernández et la banalité de la mort}

Mais qu'en est-il par exemple des représentations de cette violence croissante dans les productions culturelles de la région ? Comment cette présence inévitable de la mort dans 
la quotidienneté des rues centre-américaines est-elle transposée dans le texte littéraire ? Un cas qui nous intéresse de manière particulière est celui de l'écrivaine salvadorienne Claudia Hernández, qui, dans sont recueil de nouvelles De fronteras nous offre une série d'images perçantes de la réalité des violences urbaines dans le contexte centre-américain. De fronteras a été publié au Guatemala en 2007 et se compose d'une série de seize nouvelles courtes, lesquelles présentent un décalage important avec la réalité puisqu'elles mélangent des éléments fantastiques ou surnaturelles.

L'écrivaine salvadorienne nous présente dans son ouvrage une série de portraits hallucinés, surréalistes ou ironiques en rapport avec une quotidienneté surprenante. Dans ces histoires désenchantées nous remarquons de manière particulière une rencontre directe avec la mort dans la matérialité du cadavre représenté dans une société de plus en plus habituée à la confrontation avec la violence. Avec le ton précis de l'humour noire, l'auteure nous fait plonger dans une société qui se trouve insensibilisée face à la force soudaine de la violence, à une société qui ne se surprend guère de la torture, des disparitions ou des assassinats. Une des premières questions que l'on peut se poser à partir de la lecture des novelles d'Hernández est celle du rapport à la mort dans une société qui se déchire à cause de la banalisation du crime. Il s'agit d'un exemple parmi d'autres d'une caractérisation de plus en plus courante dans les productions culturelles centre-américaines et notamment dans la littérature contemporaine de la région.

11 Afin de mieux comprendre les stratégies d'écriture d'Hernández nous allons nous référer à un extrait de la dernière nouvelle du recueil, « Manual del hijo muerto » :

Cuando el hijo está en forma de trozos

Causa especial conmoción reconstruir el cuerpo del niño (24-25 años) que salió completo de la casa hace 2 o seis días. Por tal razón, se recomienda tener a mano una caja de pañuelos desechables y no fumar durante el proceso, a fin de evitar humedecer o dañar con fuego y cenizas las delicadas piezas. Antes de iniciar la labor, se sugiere además cerciorarse de que cada una de las partes que le han sido entregadas se corresponda con las señas particulares de su hijo y ensamblen armoniosamente. Con frecuencia el reconocimiento puede realizarse a simple vista, pero no está de más comparar la dentadura del cadáver con las placas registradas en el archivo del dentista de la familia. (Hernández, 2007: 107)

«Manual del hijo muerto » se présente comme une sorte de manuel d'instructions, grâce au caractère impératif des actions à suivre, et dans l'organisation de la mise en page du texte. Dans ce dernier texte du recueil, les éléments typographiques marquent une différence avec les nouvelles qui le précèdent puisqu'il prend effectivement la forme d'un texte typiquement non littéraire. L'indication en haut de la page signalant une numérotation distincte à celle du recueil de nouvelles laisse comprendre qu'il s'agit d'un court d'extrait d'un manuel. Comme dans toute sorte de manuel d'instructions, le lecteur est face à un problème quotidien ; nous retrouvons ainsi un style simple avec des encadrés et des notes explicatives afin de guider le lecteur dans sa démarche particulière, en l'occurrence recoller les morceaux d'un fils mort. Nous pouvons observer dès le titre et le sous-titre une rupture importante non seulement avec le style formel de la nouvelle, mais aussi avec la réalité. Cette rupture a été interprétée comme une sorte de littérature fantastique ${ }^{7}$ dans la mesure où la réalité est déformée pour dépeindre une société complètement cynique face au crime et à la violence. À notre sens, plus qu'une réécriture du fantastique, nous pouvons interpréter les procédés narratifs utilisés comme une hyperréalité, une réalité poussée à l'extrême de la violence qui se trouve à la base de son organisation.

13 Il est possible de distinguer cette rupture puisque dans le texte il n'est pas question de crime, viol, douleur, ou même justice ; l'intérêt principal est développé dans le besoin de présenter un cadavre à peu près normal à la famille et aux amis. Le décalage évoqué plus haut dans les situations décrites se matérialise dans les priorités consignées par le manuel, c'est-à-dire recoller les morceaux d'un cadavre de sa propre famille pour qu'il puisse donner une image paisible. Le lecteur doit donc assumer que l'effet choquant de la violence de cette scène ne fonctionne pas de la même manière dans l'univers du récit. Par exemple dans un des encadrés explicatifs nous pouvons lire : «Atención : atender estas medidas de precaución puede evitarle 
un desgaste innecesario en el caso de que le hayan entregado un hijo equivocado » (Hernández, 2007: 107), ce qui implique une quantité considérable de meurtres par habitant. Nous pouvons ainsi en déduire que la seule possibilité de concevoir un manuel avec ce genre d'instructions implique un contexte dans lequel la violence se banalise jusqu'au point de devenir non seulement quotidienne, mais aussi dépourvue complètement de sentiments et de toute sorte de revendication de justice sociale.

Le citoyen auquel est adressé le manuel ne peut et ne doit pas faire allusion à aucune sorte d'explication de la mort du fils. Il doit se tenir seulement au fait d'être en possession de son cadavre et d'en faire hommage. Selon les instructions données il ne faut surtout pas chercher les coupables :

Preste especial atención a las manos y pies. Estos suelen — si uno se fija muy bien — revelar escenas del padecimiento pre-muerte del hijo en cuestión. Para evitar hundirse en la tentación de elaborar hipótesis y encontrar culpables mediante las señas que dejan, cúbralos con guantes y medias de algodón oscuros. [...] Esparza sobre el rostro una capa considerable de maquillaje - colores a tono con la tez — para disimular los golpes que posiblemente presente. (Hernández, 2007: 109)

Finalement, après avoir suivi ces pas essentiels, le fils est prêt à être présenté publiquement pour les funérailles. Cependant, il est évident dans la citation précédente que la banalisation de la violence et le manque de douleur devant la représentation d'un cadavre dépecé ne cache pas complètement les raisons et le contexte de la mort, car la famille en est consciente mais elle ne peut rien y faire. Le silence s'impose. C'est ici que touts les non-dits résultent hautement signifiants dans la compréhension de la banalisation de la mort : le fait de dissimuler les marques témoignant de la violence met en évidence un système incapable de rendre justice aux victimes, dans lequel la dénonciation des faits est infructueuse et la recherche de vengeance complètement inutile. L'expression « el padecimiento pre-muerte » se présente comme un euphémisme de l'abatage humain, laissant ainsi penser le meurtre calculé comme une condition naturelle. Le cadavre, preuve matérielle de la mort, devient un objet quotidien qui se monte et se présente tel un meuble d'après son manuel d'instructions.

Nous sommes dans le texte littéraire face à une société poussée à l'extrême en ce qui concerne une représentation de la mort dénouée des rituels traditionnels judéo-chrétiens des pays latinoaméricains, mais surtout désensibilisée et ostracisée. Nous pouvons comprendre en tant que lecteurs une idée sous-jacente d'acceptation d'un système corrompu où il vaut mieux ne pas poser des questions : c'est à notre sens la réification du cadavre qui véhicule de la meilleure manière une critique poignante au manque d'action de la société civile et du gouvernement face à la violence. Les stratégies narratives mises en place dans le texte d'Hernández — telles que la fracture avec la réalité et l'humour noire de certaines situations absurdes — dépeignent avec ironie l'évolution possible d'une société marquée par la violence extrême.

Nous retrouvons une autre variation sur le même thème du cadavre comme objet quotidien dans deux autres nouvelles du même recueil intitulées Hechos de un buen ciudadano, présentées comme une histoire en deux volets. L'intrigue commence lorsque le narrateur rentre chez lui et retrouve un cadavre de femme gisant par terre dans la cuisine. La surprise du narrateur ne va pas au-delà du fait que les lieux étaient très propres, sans une trace de sang ; le corps en soi ou le fait qu'il se retrouve dans sa maison ne causent pas de consternation. Il affirme avoir déjà vu de nombreux cadavres dans sa vie. La première surprise pour le lecteur est la façon d'agir du protagoniste en bon citoyen, puisqu'il publie une annonce dans la presse afin de retrouver le propriétaire du corps :

Como cualquier buen ciudadano habría hecho, no esperé a que apareciera mensaje alguno en la radio o en la televisión, sino que hice imprimir uno en el periódico que decía:

Busco dueño de cadáver de muchacha joven de carnes rollizas, rodillas saltonas y cara de llamarse Lívida. Fue abandonada en mi cocina, muy cerca de la refrigeradora, herida y casi vacía de sangre. Información al 271-0122. (Hernández, 2007: 17)

Les appels téléphoniques ne se font pas attendre. Cette fois les effets de la violence ne sont pas dissimulés, deux personnes intéressées contactent le narrateur car ils ont perdu l'un de leurs proches, une troisième souhaite féliciter le protagoniste car « ya no hay personas como usted » 
dit-elle. Et une dernière s'intéresse aux mesures de salubrité prises concernant le cadavre. Un élément met en commun les réactions des intéressés ainsi que celle du protagoniste : il n’y a pas d'étonnement, surprise ou indignation face aux effets de la violence représentés par la femme morte dans la cuisine. Les lecteurs de l'annonce souhaitent retrouver leur parent en utilisant leurs propres moyens, et il paraît normal de répondre à une petite annonce, car des cadavres on peut en trouver par tout. Ce couple cherche une femme qui s'appelle Lívida et qui devrait être vivante, correspondant très bien à la description du cadavre. Néanmoins le narrateur, voulant leur donner encore de l'espoir, n'a pas rendu le cadavre. Toujours dans l'idée d'être un citoyen exemplaire, le narrateur décide de donner le cadavre à la famille qui cherche un corps « frais » d'homme, de cette manière ces derniers pourraient faire leur deuil - tout en laissant le cercueil fermé pour ne pas dévoiler la ruse - et les autres pourraient rester confiants d'un jour retrouver leur enfant vivante. C'est ainsi que le protagoniste se place dans son récit comme un homme exemplaire, un bon citoyen, puisqu'il considère avoir résolu la question encombrante du cadavre.

Cependant, plus loin dans le recueil de nouvelles nous retrouvons la suite de l'histoire dans la deuxième partie. Cette fois, le protagoniste se voit assailli d'appels d'autres personnes qui souhaiter connaître la façon de solutionner ce type de problème car ils ont eux aussi retrouvé des cadavres. Son appartement devient une sorte de centre d'accueil pour les personnes concernées qui apportent à leur tour une vingtaine de cadavres retrouvés. Les actes citoyens continuent en instruisant les gens dans la conservation des corps et dans la rédaction d'annonces pour la presse. Cette sorte d'atelier aboutit à l'identification des familles des treize cadavres. La cause commune dans le but de résoudre un problème social rassemble les voisins autour des discussions agréables et du partage, tout en attendant les appels des familles :

La espera fue agradable. Ellos llevaron té, café, galletas y otras bebidas y bocadillos para acompañar la conversación. La pasamos muy bien. Intercambiamos historias, algunos obsequios, ánimos y, por supuesto, alegrías cuando comenzamos a recibir las llamadas de los familiares de los cadáveres. (Hernández, 2007: 40)

De la même manière que le caractère jovial de la réunion cause la surprise du lecteur, celleci est aussi provoquée par la joie des familles retrouvant enfin « leur » cadavre. Cela marque une fois encore le décalage humoristique avec la réalité de douleur et de violence :

Trece de los veinte muertos fueron reclamados. De todas partes de la ciudad y hasta de fuera de ella aparecían parientes emocionados que nos agradecían con lágrimas por el buen trato que habíamos dado a sus muertos (algunos dijeron incluso que ni en vida habían sido tan bien cuidados sus hijos, hermanos, esposas, padres o amigas). A medida que iban siendo reclamados los cadáveres, quienes los habían encontrado se marchaban felices a sus casas. (Hernández, 2007: 40-41)

Le procédé discursif utilisé dans les trois novelles déplace la question de la violence complètement pour mettre l'accent sur d'autres problèmes beaucoup moins urgents ou qui n'ont pas la même portée dans l'imaginaire des lecteurs contemporains, telles que les rapports de voisinage et le service à la cause commune. Dans Hechos de un buen ciudadano la problématique du cadavre retrouvé se centre sur une question à la fois sanitaire et d'objet perdu, puisque le but ultime est de redonner la joie aux familles qui récupèrent un cadavre disparu et non pas de mettre en exergue le fait que l'on risque sa vie en sortant de chez soi. C'est justement ce déplacement ironique qui crée l'effet d'humour dans le texte en accordant la valeur centrale et l'attention à des tracas de la vie quotidienne. C'est dans ce jeu discursif que le cadavre devient un objet quotidien, encombrant, problématique, dont il faut savoir disposer dans les règles d'un bon citoyen. La cible du procédé ironique serait donc la violence banalisée, celle qui perd sa force constitutive et impactante de violence subjective pour se représenter comme un élément constitutif de la quotidienneté avec le niveau de gravité que cela comporte. La façon dont le narrateur gère le problème des cadavres constitue dans ces deux nouvelles la rupture narrative ouvrant la voie à une hyperréalité qui déplace la situation de violence.

Cependant, les bonnes actions du citoyen modèle ne sont pas encore finies, il reste encore le problème de sept cadavres non réclamés. Pour le narrateur, la solution se trouve de toute évidence dans le bien commun, c'est pour cela qu'il a décidé de dépecer les corps, de les faire bouillir et de préparer par la suite une soupe avec la chaire humaine : El sabor era inmejorable. 
Estaba yo seguro de que gustaría, así que llevé el guiso a los sitios que albergan pordioseros, indigentes y ancianos y les serví abundantes porciones las veces que desearon (Hernández, 2007: 41).

Cet acte de charité a été célébré par la ville entière, déclarant ainsi le narrateur « hombre bueno y ciudadano meritísimo ", toujours en accord avec les valeurs montrées dès le début du récit. Une fois encore, le lecteur se retrouve face à un décalage important qui organise la stratégie narrative et ironique dans le texte : les mérites du citoyen exemplaire qui effacent les implications de la violence extrême. Il s'agit justement de cet effacement de la force violente des faits racontés qui est mis en avant à travers le récit des annonces des cadavres, puisque leur quotidienneté les rend invisibles, dépourvus de toute importance pour la société.

\section{Conclusions}

Il est indispensable à notre sens de se référer au contexte centre-américain contemporain, à la perception de la violence et à la peur des habitants, en particulier ceux de la partie nord de la région, pour mieux comprendre les stratégies narratives développées dans les nouvelles de l'écrivaine Claudia Hernández. La rupture créée grâce à l'ironie dans l'histoire racontée se montre comme un exemple des représentations contemporaines de la mort dans une région souffrant les conséquences d'une déchirure sociale importante. L'ironie des nouvelles trouve son origine non pas dans un simple procédé stylistique, mais dans un fonctionnement des sociétés violentes, dans lesquelles les gestes quotidiens s'adaptent à l'agressivité ambiance. Ici la mort ne représente pas le même tabou prédominant dans d'autres sociétés qui la montrent d'une manière très lointaine et aseptisées ; c'est ainsi que les effets sur les lecteur dépendent à mon avis du cadre de référence dans lequel il se situe, ce qui produit soit l'effet comique soit l'horreur face à la mort banalisée. Les cadavres retrouvés dans le caniveau dans le film documentaire de Christian Poveda par exemple ou les représentations violentes dans la littérature d'Horacio Castellanos Moya font écho à cet univers fortement ironique présenté par Claudia Hernández dans De fronteras. Le cadavre comme synecdoque de la mort devient dans le récit un objet égaré et récupéré, un objet qui porte en soi le poids d'une société qui ne peut plus se préoccuper autant de la mort lorsque celle-ci se retrouve dans chaque coin de rue, insouciante et injuste. La question qui reste est sans doute jusqu'où peut aller la naturalisation d'un phénomène de violence sociale dans la vie quotidienne, pour que celle-ci n'empêche la réflexion et le droit à la justice?

\section{Bibliographie}

Banco Mundial, Crimen y violencia en Centroamérica, un desafío para el desarrollo, Guatemala : Departamentos de Desarrollo Sostenible y Reducción de la Pobreza y gestión Económica, Región de América Central y el Caribe, 2011.

Cuevas Molina, Rafael, «Tendencias generales del arte y la literatura en la Centroamérica contemporánea », in Suplemento Cultural, $\mathrm{n}^{\circ}$ 091, Instituto de Estudios Latinoamericanos.

Haas, Nadine, « Representaciones de la violencia en la literatura centroamericana », in GIGA working papers, Allemagne : German Isnstitut of Global and Area Studies, $\mathrm{n}^{\circ}$ 148, 2010.

Hernández, Claudia, « Hechos de un buen ciudadano I », in De fronteras, Guatemala : Editorial Piedra Santa, 2007

Pérez-Ratton, Virginia, 2011, « ¿ Qué región ? Apuntando hacia un estrecho dudoso », in Istmo n 22, Revista virtual de estudios literarios y culturales centroamericanos, enero-junio 2011.

Rojas, José Pablo, «Hechos de un buen ciudadano de Claudia Hernández : la naturalización de lo fantástico », in Kañina Revista de Artes y Letras, San José : Universidad de Costa Rica, XXXVIII (1), 2014.

Vergara Heidke, Adrián, « Análisis crítico del sensacionalismo : la construcción mediática de la criminalidad en la sociedad costarricense », in Iberoamericana, VIII 32, 2008, Pittsbourgh.

\section{Documentaires}

Poveda, Christian, La vida loca, Mexique-France, 2009, 90 min.

Dewer-Plana, Miquel et Fougère, Isabelle, Alma, une enfant de la violence, ARTE France, 2012, 56 min. 
Notes

1 Pour une analyse des nouvelles tendances des arts visuelles en Amérique Central : PérezRatton, Virginia, 2011 ; Cuevas Molina, Rafael, n91.

2 Dans cette article de Adrián Vergara nous trouvons un exemple des effets du discours de la violence et la peur dans les medias (télévision) au Costa Rica : Vergara Heidke, Adrián, 2008. 3 De nombreux articles journalistiques reprennent les statistiques sur la violence dans la région centre-américaine chaque année. Voici quelques exemples : « Las estadísticas confirman violencia y pobreza en América Central » Elcomercio.com, San José, 9 de julio de 2013 : «Entre 2000 y 2011, las tasas de homicidio en los siete países del istmo casi se duplicaron, al pasar de 22 a 40 casos cada 100000 habitantes, lo que hace de América Central la región sin conflicto bélico más violenta del mundo, aseguró el trabajo de Estado de la Región ». Autres : «Persiste elevada criminalidad en Latinoamérica pese a mejoras sociales », in Elcomercio.com, Cali, 26 de junio de 2013 ; «Los tres países más violentos se encuentran en América Central » El Universal, 7 de noviembre de 2011.

4 Banco Mundial, 2011, Crimen y violencia en Centroamérica, un desafío para el desarrollo, Departamentos de Desarrollo Sostenible y Reducción de la Pobreza y gestión Económica, Región de América Central y el Caribe.

5 «Las dos maras principales de Centroamérica, la Mara Salvatrucha (MS13) y la Calle 18 tienen sus raíces en los Estados Unidos. A principios de la década de 1980, más de un millón de centroamericanos viajó a los Estados Unidos escapando de la violencia y la adversidad del conflicto civil en la región. Viviendo en la pobreza y marginado por los demás grupos, un pequeño porcentaje se involucró con las maras. Algunos se unieron a Calle 18, una pandilla principalmente mexicana que se estableció muchos años antes de la ola migratoria centroamericana, mientras que otros formaron la MS13. A mediados de la década de 1990, muchos centroamericanos, incluyendo algunos miembros de las maras, fueron deportados a sus países de origen, en donde algunos se vieron envueltos en actividades criminales y reprodujeron a las maras Calle 18 y MS13. Con el tiempo, ciertos actos violentos de alto perfil pusieron a las maras en el ojo público. Los gobiernos respondieron con la puesta en marcha de una variedad de políticas de mano dura que hicieron énfasis en la represión y la judicialización, reduciendo al mínimo la prevención, rehabilitación y reintegración social de los miembros de las maras » (Banco Mundial, $2011: 15$ ).

6 Un exemple frappant est celui du film de Christian Poveda, La vida loca (2009), documentaire poignant sur les maras au Salvador. L'engagement du réalisateur lui a couté la vie. Peu de temps avant la finalisation du film, il a été abattu au Salvador, en septembre 2009. Un autre document audiovisuel est le web documentaire coproduit par ARTE France relatant le témoignage d'une jeune fille membre d'une mara au Guatemala : Alma, une enfant de la violence (2012).

7 Voir Rojas, José Pablo, «Hechos de un buen ciudadano de Claudia Hernández : la naturalización de lo fantástico », in Kañina Revista de Artes y Letras, Universidad de Costa Rica, XXXVIII (1), 2014.

\section{Pour citer cet article}

Référence électronique

Sergio Coto-Rivel, « « Notre cadavre quotidien », représentations littéraires de la mort et de la violence en Amérique centrale », Amerika [En ligne], 12 | 2015, mis en ligne le 03 juillet 2015, consulté le 12 mai 2016. URL : http://amerika.revues.org/6372 ; DOI : 10.4000/amerika.6372

\section{À propos de l'auteur}

Sergio Coto-Rivel

Université de Nantes 
s.coto-rivel@hotmail.fr

\section{Droits d'auteur}

(C) Tous droits réservés

\section{Résumés}

La littérature centre-américaine contemporaine montre un intérêt particulier par les différentes manifestations de la violence croissante dans l'Isthme, ses formes, ses bourreaux et ses victimes. Les représentations de la mort sont ainsi abondantes dans l'œuvre des écrivains contemporains qui reviennent sur les faits historiques pour la construction de la mémoire ou pour dénoncer la peur qui s'installe dans la région. Toujours dans cet esprit, le recueil de nouvelles De fronteras de l'écrivaine salvadorienne Claudia Hernández présente une série des portraits hallucinés des formes diverses de violence, mais aussi de la banalisation de la mort dans une société absorbée par une criminalité excessive. Dans cet article, après une mise en contexte de la situation centre-américaine des dernières années, nous analysons les stratégies narratives dans 3 nouvelles d'Hernández, dans lesquelles le cadavre en tant qu'objet banalisé se montre con un élément clef de la construction du récit et de la critique poignante aux sociétés centre-américaines contemporaines.

Contemporary Central American literature shows a specific interest through the different expressions of an increasing violence in the Isthmus, its forms, its persecutors and its victims. The portrayals of death abound in the works of modern writers who revisit historical facts in order to build a memory or to denounce the settling of fear within the region. In the same spirit, the collection of short stories De fronteras, by Claudia Hernández, a Salvadorian writer, depicts a set of distraught portraits of the various forms of violence, so much as the trivialisation of death in a society soaked in immoderate criminality. In this article, after contextualising the situation of Central America in the past few years, we will analyse the narrative strategies in three of Hernández's short stories in which the corpse, as a commonplace object, is a key-element to the story construction and to the mournful critique of contemporary Central American societies.

\section{Entrées d'index}

Mots-clés : violence, mort, littérature centre-américaine, Salvador, nouvelles, Claudia Hernández, criminalité

Keywords : violence, death, Central American literature, Salvador, short stories, Claudia Hernández, criminality 